\section{Unzureichende Screeningprogramme}

Viele Länder haben inzwischen den potenziellen Wert von Krebsscreeningprogrammen erkannt. Weltweit gibt es allein 43 verschiedene Programme für das kolorektale Karzinom. Doch die Qualität ist zum Teil erschreckend.

\begin{abstract}
$\mathrm{D}$ as vor fast zehn Jahren gebildete „International Colorectal Cancer Screening Network“ soll Screeningprogramme zum kolorektalen Karzinom evaluieren, um international möglichst valide und sinnvolle Programme anbieten zu können. In diesem Rahmen wurden nun Daten von 26 bereits bestehenden, voll etablierten Programmen sowie von neun Pilotprogrammen aus zusammen 24 Ländern erfragt. Nur drei davon konnten alle gewünschten Werte liefern - Pilotprogramme häufiger als etablierte Programme. Auch Deutschland gab nicht immer ein gutes Bild ab: So konnte das deutsche Programm zwar die Raten entdeckter Polypen (327,1 pro $1.000)$, Adenome (212,5 pro 1.000) und Karzinome (9,9 pro 1000), aber nicht die Zahl positiver Hämoccult-Tests unter allen Tests und auch nicht die Zahl an Endoskopien pro positivem Test berichten.
\end{abstract}

Die Größe der Programme unterschied sich erheblich: Manche richteten sich nur an ein paar tausend Menschen (wie etwa in der Region Rotterdam), andere an 36 Millionen (wie in Japan, wo bevölkerungsweit ab 40 Jahren getestet wird). Zu den besonders großen gehört auch das deutsche Programm mit einer Zielgruppe von 28 Millionen Menschen über 50; es ist zudem das mit Abstand älteste aller Programme (seit 1971).

Die meisten Programme nutzen den Hämoccult-Test für das primäre Screening, etwas häufiger die Guajak- als die immunchemische Methode. Letztere hatte insgesamt höhere Positivraten.

Die Detektionsraten von Polypen und Neoplasien waren bei jenen Programmen wie erwartet substantiell höher, die für das erste Screening ein endoskopisches Verfahren nutzen. Auch bei den Hämoccult-Tests gab es aber starke Un- terschiede: Dies ist wahrscheinlich zum Teil auf die Verwendung besonders sensitiver Tests zurückzuführen, wie dies etwa in Kroatien mit seiner Detektionsrate von 9,2\% und in New York (7,1\%) der Fall ist. In Spanien (1,1\%) und England $(1,7 \%)$ dagegen scheint es stringentere Kriterien dafür zu geben, wann ein Testergebnis als positiv definiert wird.

Fazit: Der zum Teil schlampige Umgang mit den Programmen ist erschreckend. Was bei Pilotstudien noch gut funktioniert, scheint in der Routine des laufenden Programms oft vergessen zu werden. Das Netzwerk hofft, dass auch die etablierten Programme in Zukunft ihre Definitionen verbessern und ihr Ergebnisse evaluieren, um die Screening-Qualität zu verbessern. „Screeningprogramm haben die Pflicht, sicherzustellen, dass sie den Teilnehmern einen Nutzen bringen“, schreiben sie.

Christina Berndt

Benson VS et al. Toward standardizing and reporting colorectal cancer screening indicators on an international level: The International Colorectal Cancer Screening Network. Int J Cancer. 2012;130(12):2961-73.

\title{
Besseres Screening für das Kolorektalkarzinom?
}

\section{Längst gibt es Konkurrenz für den klassischen Hämoccult-Test. Aber nützen die moderneren Methoden etwa der flexiblen Sigmoidoskopie auch etwas im Hinblick auf Mortalität und Inzidenz des kolorekatalen Karzinoms?}

B eim Hämoccult-Test ist klar, dass Mortalität und Inzidenz des kolorektalen Karzinoms dadurch gesenkt werden. Wie aber steht es um das endoskopische Screening mit flexibler Sigmoidoskopie oder Kolonoskopie? Es sollte eigentlich sensitiver sein als die Stuhltests, um adenomatöse Polypen zu finden.

Drei große europäische Studien erbrachten sehr widersprüchliche Ergebnisse in dieser Frage. Demnach führte ein Screening mittels Sigmoidoskopie in Großbritannien zu einer signifikant reduzierten Inzidenz des kolorektalen Karzinoms um $23 \%$ sowie eine nichtsignifikante Reduktion der Mortalität um $31 \%$. In Italien ergab sich eine um $18 \%$ reduzierte Inzidenz und eine um $11 \%$ gesenkte Mortalität. In Norwegen aber zeigte sich nach einem Follow-up von sieben Jahren kein Nutzen für die Sigmoidoskopie.

Eine umfassende Studie hat nun den Effekt des Screenings erneut untersucht: 1993-2001 wurden 154.900 Männer und Frauen zwischen 55 to 74 Jahren randomisiert, entweder eine flexible Sigmoidsokopie (wiederholt nach 3 oder 5 Jahren) oder die übliche Behandlung zu erhalten. Von den 77.445 Teilnehmern, die dem Screening zugelost wurden, ließen $83,5 \%$ direkt eine flexible Sigmoidoskopie vornehmen; 54,0\% wurden nach 3 oder 5 Jahren noch einmal untersucht.

Nach median 11,9 Jahren war die Inzidenz des kolorektalen Karzinoms in der Interventionsgruppe 11,9 pro $10.000 \mathrm{Per}$ sonenjahre, in der Kontrollgruppe 15,2 (relatives Risiko [RR] 0.79; $\mathrm{p}<0,001$ ). Die Reduktion war sowohl bei distalen als auch bei proximalen Tumoren signifikant ( $R R=0,71$ bzw. 0,86). Die Zahl der Todesfälle betrug in der Screeninggruppe 2,9, in der Kontrollgruppe 3,9 pro 10.000 Personenjahre. Die Mortalität durch distale Tumoren wurde durch das Screening um $50 \%$ reduziert, bei proximalen Tumoren blieb sie unbeeinflusst.

Fazit: Das Screening mit flexibler Sigmoidoskopie war mit einem signifikanten Abfall in der Inzidenz des kolorektalen Karzinoms verbunden - und zwar sowohl distal als auch proximal. Beim distalen Karzinom, das zu entdecken Aufgabe des Sigmoidskopie ist, wurde auch die Mortalität gesenkt. Einem Kommentator zufolge kommt es beim Screening aber nicht nur auf die Effektivität der Methode an, sondern auch auf die Vorlieben der Menschen: Der beste Test ist der, den sie auch machen. Christina Berndt

Schoen RT et al. Colorectal-cancer incidence and mortality with screening flexible sigmoidoscopy. N Engl J Med. 2012;366(25):2345-57. 